\title{
NEGOCIACIONES DE PAREJA: LOS TRABAJOS DOMÉSTICOS, LA CRIANZA Y LA CONSTRUCCIÓN DE LA MATERNIDAD Y LA PATERNIDAD
}

\section{Negotiations in couples: childcare, workhouse and the construction of motherhood and fatherhood}

\author{
Amaia Agirre Miguélez* \\ * AFIT, Universidad del País Vasco/Euskal Herriko Unibertsitatea \\ amaia.agirrem@ehu.eu
}

Palabras clave

Negociaciones

Trabajo

doméstico

Crianza

Paridad

Relaciones de

pareja

\section{Keywords}

Negotiations

Workhouse

Childcare work

Parity

Couple relations

\section{Resumen}

En este artículo se presentan algunas de las conclusiones de una investigación llevada a cabo con parejas que se definen a sí mismas como paritarias. Desde una perspectiva de género se analizan las negociaciones que se dan en este tipo de relaciones igualitarias. Así, se ha podido comprobar cómo los trabajos domésticos y de crianza son dos de las cuestiones que más se negocian, aunque dichas negociaciones no suelen ser explícitas ni sistemáticas, ya que se viven de manera conflictiva al entrar en contradicción con el ideal de amor romántico imperante en nuestra sociedad, que plantea la relación de pareja como una unidad en la que los intereses confluyen. De la misma manera, se ha comprobado la importancia que la construcción cultural de la maternidad y la paternidad tiene en la creación de la identidad de género y, en consecuencia, en la visión y tiempo dedicado a los trabajos de crianza.

\section{Abstract}

This article presents some of the results of a research about egalitarian couples. Specifically, the research studies the negotiations and decision-making strategies in this kind of relations from a gender perspective. Housework and childcare work are the most negotiated issues. The negotiations, however, are not explicit and systematic, because these negotiations enter in conflict with the ideal of romantic love. This ideal presents couple relation as an unproblematic union. In the same way, it has been proven the relevance of the construction of motherhood and fatherhood in the creation of gender identity and its particular implications on the vision and time spent on childcare work.

Agirre Miguélez, A., 2016, "Negociaciones de pareja: los trabajos domésticos, la crianza y la construcción de la maternidad y la paternidad", en Papeles del CEIC, vol. 2016/1, no 152, CEIC (Centro de Estudios sobre la Identidad Colectiva), Universidad del País Vasco, http://dx.doi.org/10.1387/pceic.15209 


\section{INTRODUCCIÓN ${ }^{7}$}

En este artículo se presentan algunos de los resultados obtenidos mediante una investigación sobre las negociaciones que se dan en parejas autodefinidas como paritarias en la Comunidad Autónoma del País Vasco (CAV). Estas negociaciones se desarrollan en diferentes ámbitos, como son el reparto de los trabajos domésticos y de la crianza, la sexualidad, la posesión del dinero y los recursos materiales, el tiempo y el espacio propios y el equilibrio de la relación de pareja con otras relaciones sociales y afectivas. Este artículo, en concreto, se centra en las negociaciones y el reparto de los trabajos domésticos y de la crianza, las dos cuestiones que más tiempo y reflexiones ocuparon durante las entrevistas. No obstante, aunque esto no significa que no haya otros ámbitos sobre los que las parejas analizadas negocien, como así se vio a lo largo de la investigación, al definir la relación de pareja como paritaria el reparto de estos trabajos fue la cuestión a la que continuamente se hizo referencia.

El foco de la investigación, por lo tanto, se ha puesto en la importancia que tienen las negociaciones en el camino hacia la igualdad de género en el seno de las parejas, así como en la relevancia de estas negociaciones en el ámbito del trabajo doméstico y la crianza. Estas cuestiones aparecen estrechamente ligadas, puesto que el inicio de la crianza suele ser el momento en el que se acentúan las desigualdades de género en la pareja (Schober, 2011; Abril et al., 2015), al igual que el periodo en el que aumenta el trabajo doméstico de manera muy significativa. Aún así, cada una de estas dos cuestiones tienen entidad suficiente para ser analizadas por separado, y asi es como lo expresaban las personas entrevistadas.

Como eje vertebrador de estas dos cuestiones aparecen las negociaciones de pareja, entendidas como los procesos de diálogo, las oportunidades y los mecanismos mediante los que se establecen las "reglas del juego" en las relaciones de pareja. Desde la literatura científica se ha puesto especial énfasis en las dificultades que estos procesos negociadores entrañan, ya que todavía existe un desequilibrio importante en el punto de partida de mujeres y hombres a la hora de la

\footnotetext{
${ }^{1}$ Este artículo está basado en una investigación más amplia realizada para una tesis doctoral sobre los procesos de negociación en parejas paritarias del País Vasco, dirigida por Mari Luz Esteban Galarza, y que ha contado con financiación del Vicerrectorado de Euskera de la UPV/EHU.
} 
negociación (Jónasdóttir, 1991; Langford, 1999), y es precisamente a esa cuestión a la que se acerca la investigación en la que se apoya este texto.

Aunque la idea de cuidados se haya conceptualizado más ampliamente, el artículo se circunscribe al cuidado de la descendencia, es decir, a la crianza, ya que este ámbito ha sido central en la mayoría de las entrevistas. Esto es debido a la franja de edad de las personas entrevistadas (25-50 años), que coincide con el momento vital en el que mayoritariamente se tienen hijas e hijos.

Es necesario señalar que la investigación desarrollada, y por ende, este artículo, parten desde una perspectiva de género, es decir, se considera que las relaciones de género, y las diferencias sociales que subyacen a éstas, son fundamentales a la hora de analizar y entender las relaciones de pareja en general y, más concretamente, las negociaciones y repartos del trabajo doméstico y de la crianza. Así, se pone especial atención en las diferencias socialmente construidas en torno al género, que son determinantes en las relaciones sociales.

En primer lugar se hará un breve repaso teórico de las negociaciones de pareja, seguido de una revisión de los conceptos de trabajo doméstico y crianza, y de la importancia de las ideologías de maternidad y paternidad a la hora de negociar estos trabajos. A continuación, se desarrollarán algunos apuntes metodológicos. Seguidamente se llevará a cabo el análisis de las entrevistas y, por último, se desplegarán las conclusiones más interesantes que se desprenden de este análisis.

\section{Definiendo LAS NEGOCIACIONES EN TORNO AL TRABAJO DOMÉSTICO Y LA CRIANZA: ALGUNOS ANTECEDENTES}

\subsection{Nuevos espacios familiares y procesos de negociación}

Las relaciones de pareja se han presentado como un ámbito privado, que responde a una lógica propia. En los últimos años, las prácticas han ido democratizándose, debido al proceso de individualización de sus componentes (Beck y Beck-Gernsheim, 2001; Alberdi, 1999), si bien al considerarse las relaciones de pareja como un espacio privado, esos cambios sociales no se han dado en la misma medida que en ámbitos propiamente públicos (el ámbito laboral, la participación en órganos de decisión política). Este desequilibrio entre los avances a nivel social y su 
no plasmación en el ámbito familiar conlleva conflictos y tensiones en el ámbito familiar (Cea, 2007).

La relación de pareja, aparte de poder ser un espacio para la solidaridad, también es un espacio regido por relaciones de poder, en el que se dan conflictos de intereses y en el que, mediante estrategias negociadoras, se intenta continuamente modificar el equilibrio de poder (Botia, 2010). Lo explica el hecho de que la relación de pareja está constituida por redes de cooperación y solidaridad, pero también es una institución donde pervive una clara división sexual del trabajo, que necesita de relaciones de dominación y control para su propia perpetuación (Jónasdóttir, 1991).

Al hablar de negociaciones y relaciones de poder, se tiende a unirlas automáticamente con el mundo económico, con la lucha de clases o, de manera más genérica, con las relaciones que se dan fuera del ámbito familiar, dotándolas de un carácter racional e instrumental, que se activan con el fin de conseguir algún objetivo concreto (Villarreal, 2001). Es por ello por lo que puede decirse que son relativamente innovadores los planteamientos que sitúan estas cuestiones también en el ámbito familiar y de pareja. De esta manera, se considera que las negociaciones y acuerdos también son instrumentos y realidades que vertebran las relaciones que se dan dentro del ámbito familiar.

Una de las cuestiones analizadas desde este nuevo enfoque son los cambios en las relaciones de poder basadas en las diferencias de género que se desarrollan en el ámbito familiar (Cea, 2007), y cómo éstos han dando paso a nuevas relaciones familiares y de pareja. Hoy en día puede constatarse cómo los procesos de negociación se llevan a cabo desde una posición más igualitaria respecto a décadas pasadas (Blumstein y Schwartz, 1983; Dema 2004, 2006; Hertz, 1988; Meil, 1999, 2005; Pahl, 1989). Esto provoca, paradójicamente, mayores tensiones, ya que lo que anteriormente se asumía como un "contrato natural", ya no es asumido tan claramente por ambas partes.

Aún así, en los ámbitos definidos como privados, donde las relaciones afectivas tienden a materializarse, es donde se hace más difícil ir ganando cotas de paridad y equilibrio en el poder (Dema, 2004; Díaz, 2008), lo que se traduce en un reparto desigual de las tareas y responsabilidades. En ese sentido, Capitolina Díaz sostiene que ante la aparición de un conflicto que posibilitaría la subversión del statu quo, 
tienden a prevalecer, en nombre del amor (a la pareja, a los hijos e hijas, al amor mismo...), los roles tradicionalmente establecidos (Díaz, 2008: 214-15). Así lo considera también Clara Coria (1997), que destaca la falta de negociación que existe en la mayoría de las parejas, en las que muchas cuestiones se plantean como innegociables, lo que supone un obstáculo para el desarrollo de los propios intereses, principalmente, los de las mujeres. Para esta autora, lo fundamental a la hora de iniciar cualquier negociación es tener conciencia del derecho a negociar; sin esta conciencia previa, todos los mecanismos para la negociación quedarían invalidados. Negociar en el ámbito de la pareja es fundamental para conseguir la paridad, pero, a la vez, para poder negociar con un mínimo de garantías, es necesario partir desde una situación de paridad, ya sea subjetiva u objetiva.

Otro de los obstáculos a la hora de negociar es la convicción de que en las relaciones de pareja hay ciertas cuestiones que no se negocian. Este espacio debe supuestamente estar regido por otra lógica, que tiene por objetivo complacer a los demás, muy por encima de desarrollar intereses propios, y esto es así, especialmente, en las mujeres. Es por ello por lo que en el ámbito familiar es especialmente difícil negociar, ya que explicitar estas negociaciones suele chocar con el ideal de pareja imperante (de convergencia total, de "media naranja"). Es por ello que las diferencias de criterio se plantean como algo inaceptable en una pareja bien avenida. Así, la "lógica de los afectos" (Coria, 1997: 29) dificulta la negociación dentro de las parejas, en tanto que se confunden lazos afectivos con "entrega total" y los procesos negociadores se convierten en algo problemático, ya que ponen en evidencia la individualidad de la persona frente al ideal de pareja totalmente compatible.

Por lo tanto, el primer paso indispensable a la hora de negociar es creer que los propios intereses son tan legítimos como los de la persona con la que se negocia; sentirse parte implicada de un pacto, capaz de elegir y no únicamente parte elegible (Lagarde, 2005: 453). Incluso para que las relaciones de pareja heterosexuales sean paritarias, Marcela Lagarde considera necesario que las mujeres tengan más poder que los hombres, ya que éstos cuentan con un plus de poder de partida, por lo que se hace imprescindible que las mujeres sean las depositarias de una cantidad mayor de poder desde el inicio de la relación (2005: 414). 
A la hora de analizar estas relaciones de poder, el dinero cumple un papel primordial, como lo han atestiguado diferentes investigaciones (Dema, 2004; Díaz, 2008; Nyman, 2003; Pahl, 1989). En su gestión pueden verse claramente las contradicciones que acarrean la aspiración y el discurso igualitario frente a la distribución desigual del poder, tanto material (en este caso, dinero) como simbólico. Esta distribución legitima, en muchos casos, la unilateralidad de las decisiones. Incluso en parejas que comparten un mismo estatus socio laboral y sueldo, no puede hablarse, en ningún caso, de parejas paritarias, lo que no quiere decir que se acepte pasivamente el papel subordinado y que estas relaciones estén exentas de conflictos. No explicitar estos conflictos impide una negociación abierta (Díaz, 2008: 217-23) y esto, a su vez, dificulta la consecución de una relación de pareja paritaria.

\subsection{Trabajo doméstico, cuidados y crianza}

A pesar del profundo cambio acaecido tanto en el Estado español como en el País Vasco respecto a las tasas de empleo de las mujeres, no se ha constatado un cambio tan significativo en lo que a trabajos domésticos y crianza se refiere (Durán, 1986). El aumento en la tasa de empleo de las mujeres no va parejo a una dedicación mayor a estos trabajos por parte de los hombres (Tobío, 2012). Éstos parecen quedar al margen de la nueva situación, sintiendo el derecho a gozar de una disponibilidad laboral amplia. Como consecuencia, las mujeres, en vez de conciliar, acumulan una carga mayor de trabajo, lo que se ha llamado la "doble presencia femenina" (Balbo, 1993) o "la doble jornada", que conlleva que vivan en permanente tensión y conflicto (Tobío, 2005).

El reparto equitativo de estos trabajos está lejos de materializarse, tal y como indican los datos con los que contamos actualmente (Emakunde, 2013). De manera que la forma en la que se organizan los hogares no depende únicamente del nivel de ingresos o de la disponibilidad de tiempo de sus integrantes, sino que se define y expresa en las relaciones de género que operan en esta esfera (West y Zimmerman, 1987; Coltrane, 2000). Para que esto cambie, parece imprescindible que para los hombres también exista la necesidad de conciliar la vida laboral y familiar/social (Torns y Moreno, 2008).

Así, según algunas autoras es imperiosa la necesidad de un nuevo pacto por la igualdad (Rodríguez, 2008). Mientras las mujeres reivindican su papel en la actividad pública, nadie reivindica su parte en el "espacio 
privado" al estar éste cargado de una polaridad negativa, lo que conlleva el agotamiento y la crisis del actual sistema familiar y de cuidados (Pérez, 2006). Esto, a su vez, hace más patente la necesidad de paridad tanto dentro como fuera de la esfera familiar. Esta "desafección" para con el espacio familiar y la reducida participación de los hombres en el mismo, conlleva la "repoblación" del espacio privado con mujeres que todavía se encuentran en situaciones más precarias (abuelas de avanzada edad, empleadas del hogar en situaciones precarizadas, migrantes sin derecho sociales ni laborales...), por lo que un intento de superación de unas situaciones desiguales puede crear, a su vez, otras todavía más desiguales (Tobío, 2005).

Aunque no existe unanimidad respecto al concepto del trabajo doméstico y de cuidados, podemos vislumbrar el recorrido que han tenido ambos conceptos a lo largo de las últimas décadas. En un primer momento se conceptualizaron las tareas domésticas y de cuidados como trabajo, lo que permitió visibilizar su existencia. Una vez llevado a cabo este paso, se cuantificó, lo que dejó entrever un panorama de profundas desigualdades sociales entre hombres y mujeres. Por último, se exigió una repartición justa y no basada en los roles de género tradicionales mediante el diseño de políticas públicas. Se puede ver así el amplio recorrido que ha tenido, tanto analítico como social (Torns, 2008).

Aunque estos trabajos hayan sido uno de los campos de batalla centrales desde el inicio de la segunda ola del feminismo, y una cuestión de estudio relevante desde la década de los 80 del siglo $X X$, el interés que suscitan no ha perdido vigencia, e incluso han cobrado actualidad en los últimos años al hilo de la crisis de los cuidados (Hochschild, 1995). Esta crisis ha dejado al descubierto una dimensión mucho más amplia de lo que supone el cuidado (Pérez, 2006, 2014), ya no solo centrado en la descendencia (como se consideró en un primer momento), sino también en otras personas dependientes, mayores, el auto-cuidado, la responsabilidad social del cuidado, etc. (Carrasco, Borderías y Torns, 2011; Thomas, 1993).

Otras autoras también han destacado la importancia que cobra el cuidado en la creación de la identidad femenina y masculina (en un caso por acción, y en el otro por omisión):

“El cuidado está fuertemente ligado a la identidad de género.

En una argumentación circular se ha dado por supuesto que 
las cuidadoras lo son por ser mujeres, al tiempo que son mujeres porque cuidan. La masculinidad, por el contrario, se construye como la negación de la feminidad (Maccoby 1998; Connell 2000): no ser, no actuar como las mujeres y, por tanto, no cuidar" (Tobío, 2012: 404).

En el caso del Estado español, esta revitalización en los estudios relacionados con el cuidado ha venido de la mano de "la crisis de cuidados", como ya se ha señalado. Estos estudios plantean una visión de los cuidados más amplia de la que se tenía hasta el momento (Carrasco, 2006; Pérez, 2006, 2014). Estas autoras afirman que "la crisis de los cuidados" es un problema socioeconómico de primer orden y que compete a la sociedad en su conjunto hacerse cargo de él. Así, se hace necesaria una revisión y reconfiguración de las estructuras que posibilitan que los cuidados sigan recayendo siempre sobre las mujeres, y situar la sostenibilidad de la vida como categoría analítica básica (Pérez, 2006: 8-9), por encima de las macro estructuras económicas.

A pesar de que el concepto de cuidados es muy amplio y abarca diferentes ámbitos (Torns, 2008), este artículo se centra, principalmente, en el trabajo que supone la crianza, puesto que es el tipo de cuidado que cobra mayor protagonismo en la franja de edad seleccionada para las entrevistas en profundidad.

\section{LA IDEOLOGÍA DE MATERNIDAD Y PATERNIDAD EN LA NEGOCIACIÓN DEL TRABAJO DOMÉSTICO Y DE LA CRIANZA}

La construcción cultural de la maternidad y la paternidad, y sus diferentes interpretaciones, cobra una relevancia significativa en este trabajo por su relación con los procesos de negociación que se desarrollan cotidianamente en las parejas. La maternidad se ha definido de diferentes maneras a lo largo de la historia y sin duda ha ocupado mucho más espacio y bibliografía que la paternidad o la crianza. Ha sido definida tanto como una cualidad innata en las mujeres, como una función social más o menos reconocida, o como fuente para la opresión de las mujeres (Hirata et. al., 2002: 147). Han tenido cabida, por lo tanto, diferentes visiones sociales, biológicas, políticas o económicas. Por lo que no resulta extraño que este concepto haya estado unido a consideraciones ideológicas muy determinadas, que han pretendido definirla con tintes normativos (Aguinaga, 2004). 
Los roles tradicionalmente asignados a las mujeres, con preeminencia del de madre, conllevan una identificación de la mujer con lo maternal, así como una infantilización de la misma (de la Concha y Osborne, 2004), que posibilita mantener las desigualdades de género y la subordinación de las mujeres (Firestone, 1976). Se da por hecho que las mujeres están mejor capacitadas para cuidar y relacionarse con sus hijas e hijos que los hombres, lo que posibilita crear todo un discurso normativo acerca del papel que deben cumplir las mujeres (de la Concha y Osborne, 2004). Este discurso infantilizador hace posible reducir el papel de las mujeres a una única experiencia potencial, como es la maternidad, y legitimar su expulsión de otras esferas de la vida (productivas, sociales, políticas...).

La división tradicional de roles basada en el género, y la férrea separación entre lo que se consideraba el espacio privado y el público, ha traído como consecuencia que la maternidad comprenda muchas más funciones de las que comprende la paternidad. Junto con esto, la revalorización de la maternidad que se llevó a cabo en los años 70 del siglo XX desde algunas perspectivas feministas (Rich, 1996), infravaloraba el papel que podría llegar a desempeñar la paternidad.

Un concepto clave en este proceso es el supuesto instinto maternal, mediante el que se justifica un determinado tipo de maternidad (de entrega total y dedicación absoluta, lo que Hays (1998) denomina "maternidad intensiva"), que está cobrando fuerza dentro de un proceso de renaturalización de las mujeres (Badinter, 2011). Este resurgir del naturalismo presenta un tipo de maternidad determinada como el único camino posible de realización de las mujeres, y supone una involución tanto para el feminismo como para la situación de las mujeres en general (Badinter, 2011; Esteban, 2011). Esta involución se ha acelerado en los últimos años, en los que se redescubren "valores" tradicionales, tales como la maternidad, pero ahora planteándolo como ejercicio de libertad y realización (Cruz y Zecchi, 2004: 11).

Aún así, la realidad que nos encontramos hoy en día es que Europa cuenta con una de las tasas de natalidad más bajas del mundo y de la historia, cuestión que ha merecido atención a lo largo de las últimas décadas (Alberdi, 1999). Ante la muy manida explicación de la alta tasa ${ }^{2}$

\footnotetext{
${ }^{2}$ Alta respecto a otros períodos históricos, ya que en todos los países la tasa de empleo de las mujeres es inferior a la de los hombres $y$, en algunos países es significativamente menor; si bien es cierto que en el caso del Estado español se ha dado una entrada al mercado laboral muy pronunciada en, relativamente, pocos años, proceso que ha llevado
} 
de empleo femenino, hay autoras que consideran que éste no es el factor determinante, ya que las mujeres han trabajado siempre $y$, en muchas ocasiones, en peores condiciones que las actuales. El motivo principal de la baja tasa de natalidad es que en una sociedad en la que, supuestamente, se ha conseguido la paridad institucional, esa paridad no se ha trasladado al ámbito familiar, lo que supone una contradicción importante y repercute directamente en el número de hijas e hijos que se tienen (Aguinaga, 2004).

Mientras que la carga de trabajo total de las mujeres va en aumento y la exigencia de cumplir diferentes roles se amplía (Ajenjo y García, 2004; Coltrane, 2000; Dominguez-Folgueras, 2015), se pretende que se mantenga el nivel de eficacia, lo que conlleva serias dificultades. Por otra parte, la interpretación dicotómica de la separación entre el mundo laboral y el ámbito familiar ha servido para excluir a las mujeres del primero, pero ese planteamiento, en el que ambos mundos se siguen presentando como completamente diferenciados, ha cambiado, ya que ahora se exige una doble presencia a las mujeres (Balbo, 1993), algo que no se exige a los hombres. Así, uno de los momentos en los que suelen aflorar mayores tensiones entre los roles que tradicionalmente se les han asignado a las mujeres y las prácticas cotidianas es el nacimiento del primer hijo o la primera hija (Tobío, 2005; Torns y Moreno, 2008). El nacimiento de la descendencia suele ser el momento en el que más crece la asimetría de una pareja (Abril et al., 2015; Castro y Pazos, 2011; Schober, 2011). En este momento se establecen rutinas y acuerdos que perdurarán en el tiempo, y en la medida en la que estos acuerdos no se explicitan, suele ser muy difícil cambiarlos a posteriori, lo que dificulta a su vez subvertir la división de trabajos basada en los roles de género (Solé y Parella, 2004). De esta manera, el cuidado de las criaturas aparece claramente generizado y vertebra las diferencias entre "lo masculino" y "lo femenino".

Si bien es cierto que ha habido avances al respecto (Alberdi y Escario, 2007), todavía es novedoso que los hombres cuiden. Por una parte, no saben, debido a la falta de socialización en el cuidado, que sí han tenido las mujeres. Por otra parte, no pueden, por la amplia jornada laboral y la falta de legitimidad que tienen para ejercer el cuidado, por eso Costanza

más tiempo en otros países europeos. En la CAV, la tasa de ocupación femenina en 2014 (publicada en abril de 2015, la última disponible hasta el momento) es de 44,2\%, mientras que la masculina se sitúa en 53,6\% (EUSTAT-Instituto Vasco de Estadistica, 2015). 
Tobío destaca la importancia del permiso de paternidad no transferible. La tercera razón por la que los hombres no suelen cuidar es porque no quieren, razón que está íntimamente relacionada con la construcción de la identidad de género (Tobío, 2012: 413-14).

Por todo esto, los estudios acerca de la paternidad y la corresponsabilidad en el cuidado de las hijas y los hijos no son tan abundantes como los que se ocupan únicamente de la maternidad. Aún así, hay algunos, principalmente de países anglosajones, que nos dan las claves de la implicación paterna en el cuidado de menores. Así, queda patente que el que los dos progenitores tengan un empleo contribuye a un reparto mayor del trabajo (Schober, 2011; Snarey, 1993), si bien, en ningún caso llega a ser una relación paritaria. También observan cómo el tiempo que los padres dedican a sus hijas e hijos se incrementa en la medida en la que crecen, siendo los primeros meses de vida el período en el que menos se implican (Esteban, 2011). En estos primeros meses, son las madres las que se encargan de los cuidados primarios que reciben los bebés. También se encuentran diferencias entre las actividades que llevan a cabo unas y otros. Mientras los padres invierten más tiempo en actividades recreativas o académicas, las madres, fundamentalmente, se encargan de los cuidados primarios básicos, como higiene y alimentación (Abril et al., 2015).

En esta misma línea, los datos acerca de las prestaciones por maternidad/paternidad dejan ver una clara realidad: entre el $95 \%$ y el 98\% de estos permisos lo solicitan las mujeres (Emakunde, 2011). Si bien es cierto que la prestación por maternidad solo hace referencia a las primeras 16 semanas de las criaturas, podemos suponer que esto sentará un precedente de lo que luego será un reparto sexual de las tareas que requiere la crianza, lo que se comprueba con el porcentaje desigual en las excedencias y reducciones de jornada solicitadas posteriormente.

\section{Apuntes METOdOLÓGicos}

El planteamiento metodológico de la investigación en que se apoya este texto ha sido cualitativo. Entre las técnicas disponibles se ha escogido la técnica de entrevistas en profundidad. Así, la utilización de esta técnica permite adentrarse en los entresijos mediante los que el sistema de género opera en la vida de estas personas $y$, concretamente, respecto a 
la negociación y la corresponsabilidad del trabajo doméstico y la crianza. De la misma manera, la entrevista en profundidad es una de las técnicas más utilizadas en las investigaciones sociales llevadas a cabo desde una perspectiva de género (Oakley, 1985).

Con el fin de analizar los procesos de negociación sobre el reparto de los trabajos domésticos y de la crianza, se ha entrevistado individualmente a los miembros de quince parejas. El ámbito geográfico en el que se centra es la Comunidad Autónoma del País Vasco (CAV). En cuanto a la distribución geográfica de las parejas, es la siguiente: ocho son de Gipuzkoa, seis de Araba y dos de Bizkaia. Aunque en la CAV las diferencias entre entornos urbanos y rurales no son tan significativas como en otros lugares, cabe destacar que once de las parejas residían en entornos urbanos y cuatro en rurales ${ }^{3}$.

Entre las parejas, hay once formadas por un hombre y una mujer, tres la forman dos mujeres y una la forman dos hombres. En cuanto a la descendencia, seis de las parejas tienen descendencia en común, y en otras dos parejas uno de los miembros tiene hijas y/o hijos que no comparte con la pareja actual, siete no tienen descendencia (aunque una pareja estaba acudiendo a técnicas de reproducción asistida en el momento de las entrevistas). Las edades de las personas oscilan entre los 25 y los $50^{4}$ años, y todas tienen una relación de pareja de al menos dos años de duración en el momento de la investigación. Las entrevistas se realizaron a lo largo de los años 2010, 2011 y 2012.

Las personas han sido seleccionadas mediante la técnica de "bola de nieve" (Valles, 1997: 215). Comparten un ideal paritario o feminista y, en la mayoría de los casos, cuentan con un empleo (con dos excepciones: una prejubilación y una situación de desempleo) y comparten situación

\footnotetext{
${ }^{3}$ En el entramado sociogeográfico del País Vasco se entremezcla lo rural y lo urbano, compartiendo las características demográficas más significativas que nos interesan en este estudio (número de hijos e hijas, edad al tener descendencia, tasas de empleo...). Así, la dicotomía tradicional urbano/rural en el País Vasco no tiene vigencia, porque se entremezcla estableciendo una realidad eminentemente urbana, o lo que se ha definido como rururbano.

${ }^{4}$ Se ha escogido este rango de edad de manera intencional, ya que así se puede, en primer lugar, delimitar la muestra y realizar un estudio más exhaustivo; y, en segundo lugar, resulta especialmente interesante entrevistar a personas que hayan nacido a partir de la década de 1960. Estas generaciones han vivido un cambio de sistema político-social significativo, a la par que han sido coetáneas del nacimiento y fortalecimiento del movimiento feminista en el País Vasco, por lo que resulta especialmente interesante recoger sus vivencias.
} 
socioeconómica. Así, se puede asegurar que entre las parejas seleccionadas existe un alto grado de homogamia. Este escenario favorece una eventual negociación de los trabajos domésticos y de la crianza, si bien no es suficiente pero sí conditio sine qua non para que exista una relación paritaria (Coria, 2004).

La selección de parejas paritarias, además de a factores "externos/estructurales" (como tener un empleo ambas personas...) se circunscribe también a la autodefinición: todas las personas entrevistadas se definen como paritarias o feministas o igualitarias ${ }^{5}$, aunque existen diferencias significativas que oscilan entre personas que se definen específicamente como feministas y militan en movimientos sociales, y las que se definen de una manera más laxa.

\section{PAREJAS PARITARIAS, NEGOCIACIONES Y TRABAJOS DOMÉSTICOS Y CRIANZA}

\subsection{Negociar el reparto del trabajo o cómo medir la paridad en la pareja}

Una de las cuestiones principales que destacan prácticamente todas las personas entrevistadas a la hora de analizar su definición de pareja paritaria es el reparto más o menos equitativo de los trabajos domésticos y de cuidados. Por ejemplo, así es como responde una de las entrevistadas a la pregunta de si considera que tiene una relación paritaria: "Sí, sí, sí, incluso a veces él hace más cosas que yo. Bueno, ¿qué quieres decir, en cosas de casa y así...?" (E.10.1. Mujer, 28 años, relación heterosexual). Parece residir aquí, por lo tanto, una de las formas que utilizan estas personas para "contabilizar el nivel de paridad". El reparto de estos trabajos es la forma principal de medición de igualdad, dejando de lado otras cuestiones que a priori podrían ser más polémicas o conflictivas, como son el reparto de los recursos económicos y materiales, el espacio y tiempo propios... Son estos trabajos, por lo tanto, los que centran el mayor número de negociaciones que se realizan en el seno de las parejas entrevistadas (a pesar de que esas negociaciones no se verbalicen con ese término $y$ no sean siempre explícitas $y$ sistemáticas). Por ello, estos trabajos son el ámbito privilegiado desde el

\footnotetext{
${ }^{5}$ A lo largo de las entrevistas y, por lo tanto, del análisis, estos términos se utilizan como sinónimos, sin ahondar más en su definición, puesto que es así como los han utilizado las y los participantes de la investigación.
} 
que observar cómo se desarrollan las negociaciones, acuerdos y desacuerdos.

Aunque los trabajos domésticos sean un tema constante de debate entre estas parejas, la efectividad de estas negociaciones es limitada, ya que no se hacen de una manera "sistemática y organizada", si no que suelen ser implícitas y solo se plantean abiertamente cuando hay algún conflicto que sirva de detonante. Negociar explícitamente parece ir en contra de la idea de amor romántico imperante en nuestra sociedad (Coria, 2008; Díaz, 2008), lo que resta efectividad a un posible cambio en las bases de la relación.

En el desarrollo de las entrevistas son las mujeres quiénes relatan más detalladamente los aspectos negativos que conlleva un reparto deficiente de estos trabajos, puesto que son las principales perjudicadas de la falta de equidad. Así, son ellas las que plantean reivindicaciones de cambio y alternativas: "Yo sí creo que hago más, y que a veces pasa más desapercibido porque soy una mujer, tengo como que estar todo el rato afirmando lo que hago para que se vea" (E.6.2. Mujer, 28 años, relación heterosexual). Es decir, una misma acción no tendría el mismo significado ni ocuparía el mismo espacio en la creación de la identidad de género en el caso de ser realizada por una mujer o por un hombre (West y Zimmerman, 1987).

Aún así, también hay hombres conscientes de que en sus parejas se lleva a cabo un reparto de roles de género tradicional, a pesar de ir en contra de su ideología.

No somos muy de roles tradicionales, pero a veces sí que nos repartimos los roles, yo creo. Buf... no sé cómo definirlo, a ver... No somos el hombre que va a trabajar y la mujer que se queda en casa, porque no, porque trabajamos los dos y no va en ese sentido. Pero... yo, por ejemplo, sí que me veo más en cosas de... y me fastidia ¿eh?, de, por ejemplo, se ha roto esto, y hay que arreglarlo (E.3.2. Hombre, 31 años, relación heterosexual).

Se puede afirmar que entre la mayoría de parejas entrevistadas este reparto de trabajos basado en los roles de género está en continua revisión y supervisión, puesto que las prácticas cotidianas entran en clara colisión con la ideología que se defiende, aunque es cierto que esta revisión no siempre se traduce en un cambio de prácticas (Abril et al., 2015). Hay una pareja que a lo largo de sus respectivas entrevistas 
relatan una misma anécdota que ejemplifica bien el reparto de roles tradicionales, aunque lo vivan de diferente manera:

Y no, el otro día estábamos en el sofá y ya te digo que surgió de la siesta, no sé si te lo comenté, sí, surgió, levantarse, agarrar las cosas de la lavadora y tender las cosas... y yo me quedé flipada (E.4.1. Mujer, 46 años, relación heterosexual).

Como el otro día, por ejemplo, que tiendo la ropa y me dice: gracias. No, icómo que gracias! Pero si la ropa es tuya y es mía, pues todavía le... le sale... le sale eso... (E.4.2. Hombre, 54 años, relación heterosexual).

Esta misma anécdota que aparece en las dos entrevistas la viven de manera muy diferente; mientras que la mujer se sorprende de que el hombre haya hecho algo (colgar la ropa) que considera de su responsabilidad y lo vive como una subversión de los roles de género, el hombre considera que ha hecho algo que le atañe y se sorprende, a su vez, de que la mujer le agradezca la realización de esa tarea.

Esta situación que a las dos personas les ha llamado la atención nos deja entrever cómo su ideología y sus prácticas cotidianas entran en contradicción y se crean ciertos conflictos al respecto, puesto que no siempre se corresponden sus prácticas con la imagen que tienen de la pareja misma, o de lo que debería ser una pareja paritaria a su parecer.

Este sentimiento de responsabilidad supuestamente femenina ha aparecido en diferentes entrevistas a mujeres. Así, se han recogido testimonios que relatan la disyuntiva constante entre lo que se considera que es el trabajo que les corresponde y lo que ellas creen que es justo. De esta manera, se relata la poca equidad entre las responsabilidades que asumen las mujeres y las que asumen los hombres y lo determinante que es la educación en esta cuestión:

Luego igual pasa que el día que tiene él libre no se le ocurre nunca... pues por ejemplo, limpiar el váter, o no ve nunca que el armario ese necesita no sé qué. O... yo qué sé, y yo sí que estoy ahí, yo sí lo veo, es que me han "programado" para eso, ¿no? En cierta medida sí, y a él pues no le han "programado" para eso, y eso siempre cuesta romper con eso (E.12.1. Mujer, 34 años, relación heterosexual).

Como consecuencia de la diferente socialización de hombres y mujeres, aparece también la "necesidad" de algunas mujeres de hacer las cosas "a su manera", situación que, a su vez, consideran una trampa, puesto que 
implica que ellas acaben teniendo una carga de trabajo muy superior a la de sus parejas, es decir, acaban desempeñando una "doble jornada" (Tobío, 2005):
Pero a veces caigo en esa trampa, yo quiero hacer lo que yo quiero hacer $y$ eso... hay que aceptar lo que te dan $y$ agradecer, ¿eh? Lleva todo un proceso de agradecimiento y de humildad. Todavía estoy aprendiendo... y las compras, por ejemplo, cómo las hace él, cómo organiza los gastos... a veces todavía me da el ramalazo y quiero organizar yo todo (E.9.2. Mujer, 51 años, relación heterosexual).

Por lo tanto, podemos decir que entre las personas entrevistadas no hay un intercambio de roles indiferenciado, a pesar de que sí existe un reparto informal del trabajo doméstico y de cuidados, que si bien es cierto que no es paritario, pretende, con mayor o menor éxito, acercarse a serlo. Con el término informal se quiere hacer referencia al tipo de acuerdos que se han tomado de una manera no explícita. Al no llevar a cabo negociaciones sistemáticas y no verbalizar los acuerdos, se refuerza el reparto de trabajos tradicionales; así, situaciones que a priori son puntuales o eventuales, tienden a perpetuarse en el tiempo:
No, no es costumbre, no, más o menos vamos repartiendo, oye, pues está esto, hay que hacer esto, hay que hacer lo otro, sin más, vamos. Luego van saliendo las cosas, no hay tareas específicas para cada uno ni nada por el estilo, ni un organigrama, si van saliendo las cosas, pues se hacen... (E.7.1. Hombre, 35 años, relación heterosexual).

A la hora de repartirse los trabajos domésticos y de cuidados, muchas parejas consideran que lo hacen de manera natural, lo hacen como "les sale", sin necesidad de estipular acuerdos concretos o reflexionar acerca de los mismos (Coria, 1997). Así, son minoría las parejas que sí hacen un reparto consciente y consensuado. En general, entre las personas que son más conscientes de las desigualdades de género y que tienen una ideología feminista más marcada, sí reconocen la necesidad de explicitar los acuerdos que se dan por hecho, puesto que consideran que de esta manera se observa mejor qué es un buen reparto y qué no. En cuanto a conciencia feminista, se quiere hacer referencia a personas, principalmente, que militan o han militado en movimientos feministas, que tienen una formación teórico/práctica profunda sobre las desigualdades de género y de lo que suponen. Otras investigaciones recientes señalan igualmente la importancia de "unas ideas más 
elaboradas sobre el género" a la hora de una repartición equitativa de los trabajos que suponen la crianza (Abril et al., 2015: 13).

En concreto, estas parejas que verbalizan más a menudo los acuerdos, además de diferenciarse por una mayor conciencia feminista, coinciden con que las mujeres tienen una mejor situación laboral y un mayor sueldo que sus parejas hombres, lo que apunta en la misma dirección que otras investigaciones hechas al respecto (Dema, 2004; Díaz, 2008; Nyman, 2003; Pahl, 1989). Es decir, una posición de partida favorable en las condiciones materiales, facilita la creación de una conciencia sobre la necesidad de negociar. Pero, aun siendo consciente de la necesidad de verbalizar este reparto, se encuentran resistencias a la hora de negociar:

No hacemos nada... a pesar de que yo le amenacé con ponerlo todo por escrito, de hacer una lista con lo que hace cada uno. Pero no hemos llegado hasta ahí, esto para él es un gran conflicto. Si lo escribes, en mi opinión, se ve mucho mejor, qué cosas se hacen... qué se olvida... Porque las cosas que tú haces, no se quedan registradas, pero esto ya le parece... ponerse a malas, no sé cómo decirlo. Y al final no lo hemos hecho nunca (E.12.1. Mujer, 34 años, relación heterosexual).

Como se ha mencionado en los primeros epígrafes, mediante este fragmento puede verse cómo el ideal de amor romántico dificulta la negociación en las parejas, en tanto que se confunde relación de pareja con compatibilidad total y ausencia de conflictos. Este ideal de pareja conlleva que las negociaciones explícitas se vivan como algo problemático (Coria, 1997), como parece hacerlo la pareja de esta mujer, que interpreta la propuesta de anotar las tareas realizadas por cada miembro como "ponerse a malas". Así, considerar que hay temas innegociables en los que no debe existir otra lógica más que la de los afectos o que la propia negociación en sí sea "un gran conflicto", es un claro impedimento a la hora de que las mujeres defiendan sus propios intereses. Como se ha señalado, el primer paso para defender intereses propios es considerarlos tan legítimos como los de la persona con la que hay que negociar, es decir, ser consciente del derecho a negociar. Entre las personas entrevistadas, aunque hay mujeres que sí lo consideran así $y$, por lo tanto, reclaman este derecho, son innumerables los obstáculos con los que se encuentran a la hora de materializarlo (Coria, 1997; Lagarde, 2005), como puede apreciarse en la cita anterior. 
Aparte de plantear las negociaciones de manera explícita, otra de las estrategias que utilizan algunas de las mujeres entrevistadas para hacer visible el reparto desigual es aplazar las tareas a realizar:

Yo a veces practico la resistencia pasiva [risas], hay alguna cosa que suelo hacer yo siempre, como por ejemplo la lavadora, pero que no lo hemos decidido así, si no que ha surgido y se ha quedado como está, pues... si me harto dejo de ponerla, a ver cuánto aguanta así. y mi pareja no me dice nada, claro, pero los dos nos enteramos de qué va el asunto, que le estoy mandando un mensaje (E.6.2. Mujer, 28 años, relación heterosexual).

De esta manera se "obliga" a la otra persona a que las realice y, así, además, se pone en evidencia que el reparto establecido de facto no ha sido acordado verbalmente y no es justo. En esta cita puede verse cómo el reparto de trabajos que establecía el "contrato natural" vigente en décadas pasadas (Dema, 2006) no es tan fácilmente aceptado como anteriormente y aunque esto no conlleve una negociación explícita, sí que desencadena prácticas que ponen en evidencia la necesidad de reformular ese reparto y la existencia de estrategias de renegociación conscientes.

\subsection{La negociación en la crianza o de cómo se construye la maternidad y la paternidad}

Si bien es cierto que entre las personas entrevistadas el tema central de sus negociaciones eran los trabajos domésticos, cabe señalar que no lo eran tanto los trabajos de cuidados. Al ahondar más en el reparto que se hace de estos trabajos, se ha podido constatar cómo existe una división sexual mayor en los trabajos de cuidados que en los trabajos domésticos, por lo menos, durante los primeros años de la descendencia:

Y la verdad es que, joe, estos últimos años he estado súper metida en todo el tema de los embarazos, parir, los niños [tiene una hija y un hijo con su pareja] y sobre todo yo al pie del cañón, un poco en eso, y ahora ya que se van haciendo un poco mayores me apetece salir un poco de ahi, hacer algo diferente, porque al final si no sales de ahí siempre acabas hablando de niños, haciendo cosas de niños... (E. 13.1. Mujer, 31 años, relación heterosexual).

Esta identificación de la mujer con lo maternal (de la Concha y Osborne, 2004) y el desarrollo de un único modelo de "maternidad intensiva" 
(Hays, 1998) ahonda en una mayor división sexual de estos trabajos de cuidados.

Paradójicamente, que esta división sexual sea más clara en la crianza que en los trabajos domésticos no conlleva una mayor negociación sobre los trabajos asociados a la crianza. En el reparto de estos trabajos la maternidad ocupa un lugar preeminente, no tanto la paternidad que, a pesar de que en todas las entrevistas con hombres que eran padres aparece, no lo hace con la misma intensidad que en las entrevistas con mujeres que eran madres. Esto nos da una clave para entender en torno a qué se vertebran las desigualdades en esta materia, puesto que se considera que la maternidad implica muchas más tareas que las que implica la paternidad, y es asi como lo perciben también algunas de las entrevistadas:

Parece que al tener niños florece todo eso, qué papel tiene la madre, qué papel tiene el padre... y de repente, ser madre es como... nunca lo habías pensado y de repente eres madre y ya no hay nada más en el mundo, bueno, ¿y antes qué? ¿antes eso no era así? Había otras cosas... (E.15.1. Mujer, 44, años, relación homosexual).

Así, una de las mujeres entrevistadas afirma que el cuidado de las hijas es a veces un obstáculo a la hora de desarrollar sus intereses y relata cómo vive la ambivalencia entre el miedo a dejar a sus hijas con otra persona que no sea ella, y el miedo a que eso pueda conllevar perder algunas relaciones sociales:

Y yo todavía no he salido... igual tenía que salir más... porque él sí ha salido, pero igual es que yo tengo más miedos... o más ganas de estar con la pequeña y me parece que esto es una fase, que luego ya no querrán estar conmigo y luego ya estaré con mis amigas ¿no? Pero también me da miedo perderlas, perder la relación... la confianza... (E.12.1. Mujer, 34 años, relación heterosexual).

El concepto de instinto maternal y la naturalización de la maternidad que conlleva (Badinter, 2011) comporta este tipo de contradicciones entre los intereses propios y el modelo de maternidad intensivo al que se aspira, modelo que se ha revitalizado en los últimos años (Esteban, 2011).

Mientras que los trabajos domésticos son cuestiones que se discuten o hablan más, los cuidados, sobre todo de los primeros años, no se 
negocian tanto entre estas parejas y, en la mayoría, parece existir un acuerdo tácito que otorga una responsabilidad mayor a las mujeres. Esta situación responde a la construcción de un tipo de maternidad y paternidad determinado, como se ha visto en la primera parte de este artículo.

Por ejemplo, una pareja formada por dos mujeres con dos hijos, nos cuentan cómo van turnándose a la hora de coger reducción de jornada, a pesar de saber que esta forma de organizarse conllevará serias dificultades a la hora de ascender laboralmente:

\begin{abstract}
Entonces, este año le ha tocado a Ane estar a las tardes con los niños, el año pasado estuve yo, hasta ahora lo hemos hecho así. Nadie más de la escuela lo hace así, no sé si hay muchos padres a la tarde... a la mañana sí llevando a los niños. Por ejemplo, yo creo que ahí sí que hay mucha diferencia entre la mañana, llevando a los niños, ahí sí que veo a muchos hombres, pero esos no vuelven a aparecer hasta la noche. Los niños salen a las cuatro y media y a esa hora están las mujeres, y no todas son profesoras que salgan a esa hora. Son las que renuncian a la jornada completa con todo lo que eso conlleva, porque de eso no se habla nunca, porque si tú renuncias a la jornada completa, no vas a ascender en tu trabajo, no sé... eso supone ya quedarte atrás (E.15.1. Mujer, 44 años, relación homosexual).
\end{abstract}

No es casualidad que este tipo de crianza compartida se dé en una pareja de dos mujeres. Existen investigaciones recientes que destacan que aunque en el seno de las parejas homosexuales este reparto de trabajos generizado no se dé con la misma intensidad que en las parejas heterosexuales, sí se da, en cambio, a la hora de externalizar las tareas; cuando otras personas se encargan del trabajo doméstico o de la crianza, sea de manera retribuida o no, suelen ser mujeres, principalmente las abuelas o las tías (Pichardo, 2009).

Aún asi, entre las personas entrevistadas, hay hombres que también cuidan activamente (Alberdi y Escario, 2007). Concretamente, hay dos casos en los que han sido los hombres de parejas heterosexuales los que han cogido o bien excedencia o bien reducción de jornada $y$, en un tercer caso, a pesar de plantear esa posibilidad, se le negó ese derecho en el puesto de trabajo y fue la mujer la que acabó solicitando una excedencia: 


\begin{abstract}
Ahora mismo me acabo de cambiar a otro taller, porque en el taller en el que estaba no les gustaba mi reducción, me han mandado para otro, y ahora mismo no sé, pues estoy ahí conociendo el taller nuevo y eso, pero bueno, tampoco me planteo mucho más (E.7.1. Hombre, 35 años, relación heterosexual).
\end{abstract}

En este caso en concreto, el hecho de coger una reducción de jornada le ha supuesto algún inconveniente en el empleo, como es el cambio de lugar de trabajo. Así, puede verse en esta acción no mayoritaria (Emakunde, 2011), la importancia de su significado en la deconstrucción del género (West y Zimmerman, 1987), ya que pone en evidencia los obstáculos que algunos hombres encuentran cuando llevan a cabo comportamientos hegemónicamente no masculinos, como es el cuidado de menores. Puesto que el cuidado aparece intimamente ligado a la feminidad, y la construcción de la masculinidad reside en la negación de esa feminidad y sus funciones asociadas (Connell, 2000), cuidar tambalea las bases sobre las que se asienta dicha masculinidad. Mediante este testimonio puede verse claramente la falta de legitimidad que tienen los hombres a la hora de reivindicar un papel que parece no corresponderles socialmente, tal y como plantea Constanza Tobío (2012).

Aún así, cabe destacar que en el caso concreto de estos tres hombres, son personas con un trabajo físicamente duro (en un taller, en una obra y en una fábrica) y que sus parejas mujeres son las tres profesoras, con un mayor sueldo, estabilidad laboral y un mejor horario. En el caso en el que la situación laboral es parecida para hombres y mujeres son las mujeres las que han optado por coger una excedencia y/o reducción de jornada, tal y como apuntan otras investigaciones (Abril et al., 2015).

\title{
6. CONCLUSIONES
}

Se puede afirmar que el reparto de los trabajos domésticos y de crianza es clave en las relaciones de pareja que se consideran paritarias. Aún así, sigue existiendo un reparto generizado de estos trabajos, incluso cuando la definición de paridad reside principalmente en una distribución equitativa de los mismos. Así, puede constatarse la existencia de una clara distorsión entre los discursos y autopercepciones paritarias y las prácticas cotidianas. 
Uno de los mayores obstáculos a la hora de conseguir aunar prácticas e ideología es la falta de explicitación de pactos. Explicitar pactos acerca del funcionamiento de la pareja y del reparto del trabajo que debe realizarse favorece el surgimiento de nuevos modelos de relaciones más igualitarias, del mismo modo que la menor existencia de pactos explícitos conlleva la continuidad de modelos de "pareja más tradicionales", así como la perpetuación de estereotipos de género. Si bien es cierto que todas las parejas entrevistadas creen que es fundamental llevar a cabo un reparto justo de estos trabajos para el buen funcionamiento de las mismas, no todas las personas defienden la necesidad de explicitar estos pactos o negociaciones; al contrario, defienden que el reparto que llevan a cabo se hace de manera natural, espontáneo, sin necesidad de un escrutinio continuo.

Aún así, también existen parejas que creen en la necesidad de explicitar dichos acuerdos, pero las diferencias de criterio se viven de manera conflictiva $y$, en algunos casos, se tienden a ocultar o a no hablar claramente sobre ellos, ya que negociar estas diferencias es aceptarlas, lo que entra en contradicción con el ideal de amor imperante en nuestra sociedad, que tiende a identificar el amor con afinidad total, y la disparidad de criterios con incompatibilidad.

Si bien es cierto que estos trabajos y su reparto suelen ser el instrumento con el que se "mide" la paridad en este tipo de relaciones, también es cierto que no suele haber una conversación o negociación abierta sobre su reparto, sino que suelen ir tomándose decisiones puntuales sobre la marcha y solventando los conflictos según surgen. Siempre que hay un detonante, suele plantearse la cuestión, pero sin reconstruir las bases del reparto del trabajo que posibilita estas situaciones desiguales. De esta manera, prevalecen los "acuerdos silenciosos" (Wiesmann et al., 2008), implícitos, que tienden a perpetuar este reparto desigual. Aún así, el componente de la pareja que aspira a cambiar diferentes aspectos de la misma (que en las parejas heterosexuales suele ser la mujer), es la persona que explicita más quejas o nuevas propuestas, ya que los cambios a priori le beneficiarian. Por su parte, el miembro de la pareja que se encuentra más cómodo en ella (que en las parejas heterosexuales suele ser el hombre), tiende a mostrar reticencias para negociar y llegar a nuevos pactos que cambien el statu quo. 
Por otra parte, cabe señalar que se discuten, hablan o negocian más los trabajos domésticos que los que supone la crianza, por lo menos, los primeros años de las criaturas. El ideal de maternidad intensiva está ciertamente arraigado y queda poco espacio para una crianza en igualdad, y además se exige mucho menos la intervención paterna que la materna. Entre las personas entrevistadas había tres hombres que habían solicitado excedencias y reducciones de jornada para el cuidado de menores, pero esto tenía relación directa con el tipo de empleo que desempeñaban, con peores condiciones laborales, que el de sus parejas mujeres.

\section{BibLIOGRAFÍA}

Abril, P. et al., 2015, “Ideales igualitarios y planes tradicionales: análisis de parejas primerizas en España", en Reis, 150, pp. 3-22.

Aguinaga, J., 2004, El precio de un hijo: los dilemas de la maternidad en una sociedad desigual, Debate, Barcelona.

Ajenjo, M., García, J., 2004, “Cambios en el uso del tiempo de las parejas ¿estamos en el camino hacia una mayor igualdad?", en Revista Internacional de Sociología, 72 (2), pp. 453-476.

Alberdi, I., 1999, La nueva familia española, Taurus, Madrid.

Alberdi, I., Escario, P., 2007, Los hombres jóvenes y la paternidad, Fundación BBVA, Bilbao.

Badinter, E., 2011, La mujer y la madre, La esfera de los libros, Madrid.

Balbo, L., 1993, “La doble presencia”, en C. Borderías, C. Carrasco y C. Alemany (Comps.), Las mujeres y los trabajos: rupturas conceptuales, Icaria, Barcelona, pp. 503-513.

Beck, U., Beck-Gersheim, E., 2001, El normal caos del amor. Las nuevas formas de la relación amorosa, Paidós Contextos, Barcelona.

Blumstein, P., Schwartz, P., 1983, American couples, New York, William Morrow.

Botia, C., 2010, “Una propuesta teórica para abordar las estrategias de negociación de la vida cotidiana desde la perspectiva de género", en Papers, 95/1, pp. 119-137.

Carrasco, C., 2006, "La paradoja del cuidado: necesario pero invisible", en Revista de Economía Crítica, 5, pp. 39-64.

Carrasco, C., Borderías, C., Torns, M. T., 2011, El trabajo de cuidados. Historia, teoría y políticas, Los libros de la Catarata, Madrid. 
Castro, C., Pazos, M., 2011, "Hombres, cuidados e igualdad de género. Fundamentos para la equiparación efectiva entre los permisos de padres y madres", en Congreso Iberoamericano de masculinidades y equidad: investigación y activismo, Barcelona, disponible en: http://www.redmasculinidades.com/sites/default/files/archivos/bibl ioteca/00246.pdf. Última consulta: 25/09/2015.

Cea, M. A., 2007, La deriva del cambio familiar. Hacia formas de convivencia más abiertas y democráticas, CIS, Madrid.

Coltrane, S., 2000, "Research on Household Labor: Modeling and Measuring the Social Embeddedness of Routine Family Work", en Journal of Marriage and the Family, 62, pp. 1208-1233.

de la Concha, A., Osborne, R. (Coords.), 2004, Las mujeres y los niños primero; discursos de la maternidad, Icaria, Barcelona.

Connell, R. W., 2000, The Men and the Boys, University of California Press, Berkeley.

Coria, C., 1997, Las negociaciones nuestras de cada día, Paidós, Buenos Aires.

Coria, C., 2004, El amor no es como nos lo contaron... ni como lo inventamos, Paidós, Buenos Aires.

Coria, C., 2008, "La sexuación del dinero: conflictos "subjetivos" en la "masculinidad", en la feminidad y su repercusión en la vida cotidiana de mujeres y varones", en Congreso Internacional Sare 2007 Masculinidad y vida cotidiana, Emakunde, Vitoria-Gasteiz, pp. 265279.

Cruz, J., Zecchi, B. (Eds.), 2004, La mujer en la España actual. ¿Evolución o involución?, Icaria, Barcelona.

Dema, S., 2004, La desigualdad y las relaciones de poder en el ámbito privado: análisis de la parejas con dos ingresos desde una perspectiva de género, Tesis doctoral inédita, Universidad de Oviedo, Asturias. Disponible en: http://www.tdx.cat/bitstream/handle/10803/11105/UOV0008.pdf;js essionid=DA19B95EBE6975AC4E60E57218813D19.tdx2? sequence $=1$ Última consulta: 24/09/2015.

Dema, S., 2006, Una pareja, dos salarios. El dinero y las relaciones de poder en las parejas de doble ingreso, CIS, Madrid.

Díaz, C., 2008, "La gestión del dinero en las relaciones de pareja: transiciones en los patrones de conducta y en las identidades en función del género", en Congreso Internacional Sare 2007: 
Masculinidad y vida cotidiana, Emakunde, Vitoria-Gasteiz, pp. 205223.

Dominguez-Folgueras, M., 2015, "Parentalidad y división del trabajo doméstico en España, 2002-2010", en Reis, 149, pp. 45-64.

Durán, M. A., 1986, La jornada interminable, Icaria Editorial, Barcelona.

Esteban, M. L., 2011, Crítica del pensamiento amoroso, Edicions Bellaterra, Barcelona.

Emakunde-Instituto Vasco de la Mujer, 2011, "Cifras sobre la situación de mujeres y hombres en Euskadi 2010". Disponible en:http://www.emakunde.euskadi.eus/contenidos/informacion/ser vicio_cifras/es_emakunde/adjuntos/cifras_2010_es.pdf. Última consulta: 10/09/2015.

Emakunde-Instituto Vasco de la Mujer, 2013, "Cifras sobre la situación de mujeres y hombres en Euskadi 2012. Resumen". Disponible en: http://www.emakunde.euskadi.net/contenidos/noticia/2013_05_28 _consejo_direccion/es_def/adjuntos/resumen.cifras.2012.cas.pdf. Última consulta: 24/09/2015.

EUSTAT-Instituto Vasco de Estadística, 2015, Encuesta de población con relación a la actividad, disponible en: http://es.eustat.eus/ci_ci/movil/elementos/ele0011500/ti_Tasa_de ocupacion_de_la_poblacion_de_16_y_mas_a\%C3\%B10s_de_la_CA_d e_Euskadi_por_trimestre_segun_nivel_de_instruccion_y_sexo_1_IV 2014/tbl0011537_c.html\#axzz3pCNUU9zL. Última consulta: $21 / 10 / 2015$

Firestone, S., 1976, La dialéctica del sexo, Kairós, Barcelona.

Hays, S., 1998, Las contradicciones culturales de la maternidad, Paidós, Barcelona.

Hertz, R., 1988, More Equal Than Others, University of California Press, Berkeley.

Hirata, H. et al., 2002, Diccionario crítico del feminismo, Síntesis, Madrid.

Hochschild, A. R., 1995, "The Culture of Politics: Traditional, Post-modern, Cold-modern and Warm-modern Ideals of Care", en Social Politics, Vol. 2. (3), pp. 331-345.

Jónasdóttir, A. G., 1991, Love, Power and Political Interest: towards a theory of patriarchy in contemporary western societies, University of Örebro, Örebro, Sweden.

Lagarde, M., 2005, Para mis socias de la vida, horas y HORAS, Madrid. 
Langford, W., 1999, Revolutions of the Heart. Gender, Power and the Delusions of Love, Routledge, New York.

Nyman, C., 2003, "The Social Nature of Money: Meanings of Money in Swedish Families", en Women's Studies International Forum, 26 (1), pp. 79-94.

Meil, G., 1999, La posmodernización de la familia española, Acento Editorial, Madrid.

Meil, G., 2005, "El reparto desigual del trabajo doméstico y sus efectos sobre la estabilidad de los proyectos conyugales", en Reis, 111, pp. 163-179.

Oakley, A., 1985, Sex, Gender and Society, Aldershot, Gower.

Pahl, J., 1989, Money and Marriage, Palgrave Macmillan, Basingstoke.

Pérez, A., 2006, "Amenaza tormenta: la crisis de los cuidados y la reorganización del sistema económico", en Revista de Economía Crítica, 5, pp. 7-37.

Pérez, A., 2014, Subversión feminista de la economía. Aportes para un debate sobre el conflicto capital-vida, Traficantes de Sueños, Madrid.

Pichardo, J. I., 2009, Entender la diversidad familiar. Relaciones homosexuales y nuevos modelos de familia, Edicions Bellaterra, Barcelona.

Rich, A., 1996, Nacemos de mujer; la maternidad como experiencia e institución, Ed. Cátedra, Col. Feminismos, Madrid.

Rodríguez, M., 2008, Un nuevo pacto por la igualdad, Fundación Carolina, Madrid.

Schober, P. S., 2011, "The Parenthood Effect on Gender Inequality: Explaining the Change in Paid and Domestic Work When British Couples Become Parents", en European Sociological Review, 29 (1), pp. 74-85.

Snarey, J., 1993, How fathers care for the next generation, Harvard University Press, Cambridge.

Solé, C., Parella, S., 2004, "Nuevas expresiones de la maternidad. Las madres con carreras profesionales exitosas", en RES, 4, pp. 67-92.

Thomas, C., 1993, "De-constructing concepts of care", en Sociology 27 (4), pp. 649-669.

Tobío, C., 2005, Madres que trabajan. Dilemas y estrategias, Ed. Cátedra, Col. Feminismos, Madrid. 
Tobío, C., 2012, “Cuidado e identidad de género. De las madres que trabajan a los padres que cuidan", en RIS, Vol. 70, 2, pp. 399-422.

Torns, M. T., 2008, "El trabajo y el cuidado: cuestiones teóricometodológicas desde la perspectiva de género", en EMPIRIA, Revista de metodología de las Ciencias Sociales, 15, pp. 53-73.

Torns, M. T., Moreno, S., 2008, "La conciliación de las jóvenes trabajadoras: nuevos discursos, viejos problemas", en Revista de Estudios de Juventud, 83, pp. 101-117.

Valles, M. S., 1997, Técnicas cualitativas de investigación social; revisión metodológica y práctica profesional, Síntesis Sociología, Madrid.

Villarreal, A. L., 2001, "Relaciones de poder en la sociedad patriarcal", en Actualidades Investigativas en Educación, Vol. 1, 1, pp. 1-17.

West, C., Zimmerman, D. H., 1987, "Doing Gender", en Gender and Society, 1, pp. 125-151.

Wiesmann, S. et al., 2008, “Not worth mentioning': The implicit and explicit nature of decision-making about the division of paid and domestic work", en Community, Work E Family, 11, 4, pp. 341-363. 